\title{
Characterization and optimization of the liquid crystal on silicon displays for their application to diffractive optics
}

\section{Caracterización y optimización de las pantallas LCoS para su aplicación en óptica difractiva}

\author{
Angel Lizana ${ }^{*}$,S) \\ Departamento de Física, Universitat Autònoma de Barcelona, 08193, Bellaterra, Spain. \\ (*) Email: angel.lizana@uab.es S: miembro de SEDOPTICA / SEDOPTICA member \\ Received / Recibido: 24/10/2014. Revised / Revisado: 11/11/2014. Accepted / Aceptado: 19/11/2014. \\ DOI: http://dx.doi.org/10.7149/OPA.47.4.293
}

\begin{abstract}
:
This manuscript presents a revision work related to the calibration, optimization and application of Liquid Crystal on Silicon (LCoS) displays. In particular, a thoroughly study of the LCoS displays for their application to diffractive optics is detailed. A new method of characterization and optimization of these devices is presented. This method is based on a combination of the Jones and Mueller mathematical formalisms, which is able to control and optimize the intensity and phase response of LCoS displays, even in presence of some depolarization content. Some experimental evidences of a physical phenomenon observed when working with LCoS displays are here also presented: the timefluctuations of the phase phenomenon. Related to this effect, an analysis of the influence of the timefluctuations of the phase in the performance of LCoS displays when addressing digital holograms is also presented, which has revealed that large amplitude fluctuations may significantly degrade their efficiency. Finally, a methodology for the phase encoding in LCoS displays is also proposed, which allows to minimize to certain extent the undesired influence of time-fluctuations of the phase.
\end{abstract}

Key words: LCD, LCoS, Depolarization, Phase Optimization, Diffractive optics, Phase Fluctuations.

\section{RESUMEN:}

Este manuscrito presenta un artículo de revisión en el contexto de la calibración, optimización y aplicación de las pantallas de cristal líquido sobre silicio (LCoS). En particular, se presenta un estudio exhaustivo de las pantallas LCoS, para su utilización óptima en aplicaciones dentro de la óptica difractiva. Se propone un nuevo método de caracterización y optimización de las pantallas LCoS, el cual se basa en una combinación de los formalismos matemáticos de Mueller y Jones, que permite optimizar la respuesta en intensidad y fase de estos dispositivos, incluso en presencia de cierta cantidad de luz despolarizada. También se presentan evidencias experimentales de un importante fenómeno físico que aparece al trabajar con las pantallas LCoS: las fluctuaciones temporales de la fase. Para una mayor comprensión de este fenómeno, se presenta igualmente una discusión sobre la influencia de las fluctuaciones de la fase en la eficiencia de elementos ópticos difractivos generados con las pantallas LCoS. Finalmente, también se propone un método de codificación de la fase para la implementación de elementos ópticos difractivos que permite minimizar el efecto desfavorable de las fluctuaciones de fase en la generación de hologramas digitales.

Palabras clave: LCD, LCoS, Despolarización, Optimización Fase, Óptica Difractiva, Fluctuaciones de Fase.

\section{REFERENCES AND LINKS / REFERENCIAS Y ENLACES}

[1]. H. K. Liu, J. A. Davis, R. A. Lilly, "Optical-data-processing properties of a liquid crystal television spatial light modulator", Opt. Lett. 10, 635-637 (1985). DOI

[2]. H. J. Coufal, D. Psaltis, B. T. Sincerbox, Holographic Data Storage, Springer-Verlag, Berlin (2000). DOI 
[3]. K. M. Twietmeyer, R. A.Chipman, A. E. Elsner, Y. Zhao, D. VanNasdale, "Mueller matrix retinal imager with optimized polarization conditions", Opt. Express 16, 21339-21354 (2008). DOI

[4]. A. Márquez, C. Iemmi, J. Campos, J. C. Escalera, M. J. Yzuel, “Programmable apodizer to compensate chromatic aberration effects using a liquid crystal light modulator", Opt. Express 13, 716-730 (2005). $\underline{\text { DOI }}$

[5]. A. Hussain, J. L. Martínez, A. Lizana, J. Campos, "Super resolution imaging achieved by using on-axis interferometry based on a spatial light modulator", Opt. Express 21, 9615-9623 (2013). DOI

[6]. A. Hussain, J. L. Martínez, J. Campos, "Holographic superresolution using spatial light modulator", J. Eur. Opt. Soc. Rap. Public. 8, 13007 (2013). DOI

[7]. S. Zwick, T. Haist, M. Warber, W. Osten, "Dynamic holography using pixelated light modulators", Appl. Opt. 49, F47-F58 (2010). DOI

[8]. X. Wang, W. Liu, E. Chuang, D. Psaltis, "Liquid crystal on silicon (LCOS) beam steerer for holographic data storage", Lasers and Electro-Optics 19, 407-408 (1998).

[9]. Q. Mu, Z. Cao, L. Hu, D. Li, L. Xuan, “An adaptive optics imaging system based on a high-resolution liquid crystal on silicon device", Opt. Express 14, 8013-8018 (2006). DOI

[10]. T. Inoue, H. Tanaka, N. Fukuchi, M. Takumi, N. Matsumoto, T. Hara, N. Yoshida, Y. Igasaki, Y. Kobayashi, "LCoS spatial light modulator controlled by 12-bit signals for optical phase-only modulation”, Proc. SPIE 6487, 64870Y (2007). DOI

[11]. K. M. Twietmeyer, R. A. Chipman, A. E. Elsner, Y. Zhao, D. VanNasdale, "Mueller matrix retinal imager with optimized polarization conditions", Opt. Express 16, 21339-21354 (2008). DOI

[12]. J. Galeotti, A. Sajjad, B. Wang, L. Kagemann, G. Shukla, M. Siegel, B. Wu, R. Klatzky, G. Wollstein, J.S. Schuman, G. Stetten, "The OCT penlight: In-situ image guidance for microsurgery", Proc. SPIE 7625, 762502 (2010). DOI

[13]. A. Hermerschmidt, S. Osten, S. Krüger, T. Blümel, "Wave front generation using a phase-only modulating liquid-crystal-based micro-display with HDTV resolution", Proc. SPIE 6584, 65840E (2007). DOI

[14]. A. Lizana, I. Moreno, A. Márquez, C. Iemmi, E. Fernández, J. Campos, M. J. Yzuel, “Time fluctuations of the phase modulation in a liquid crystal on silicon display: characterization and effects in diffractive optics", Opt. Express 16, 16711-16722 (2008). DOI

[15]. U. Ruiz, C. Provenzano, P. Pagliusi, G. Cipparrone, "Pure two-dimensional polarization patterns for holographic recording", Opt. Lett. 37, 311-313 (2012). DOI

[16].K. P. Proll, J. M. Nivet, K. Körner, H. J. Tiziani, "Microscopic three dimensional topometry with ferroelectric liquid-crystal-on-silicon displays", Appl. Opt. 42, 1773-1778 (2003). DOI

[17]. J. Kacperski, M. Kujawinska, “Active LCoS based laser interferometer for microelements studies”, Opt. Express 14, 9664-9678 (2006). DOI

[18]. R. M. A. Azzam, N. D. N. Bashara, "Ellipsometric measurement of the polarization transfer function of an optical system", J. Opt. Soc. Am. 62, 336-340 (1972). DOI

[19]. R. J. Gagnon, “Liquid-crystal twist-cell optics”, J. Opt. Soc. Am. 71, 348-353 (1981). DOI

[20]. K. Lu, B. E. A. Saleh, "Reducing Berreman's $4 \times 4$ formulation of liquid-crystal-display optics to $2 \times 2$ Jones vector equations”, Opt. Lett. 17, 1557-1559 (1992). DOI

[21]. A. Márquez, C. Iemmi, I. Moreno, J. A. Davis, J. Campos, M. J. Yzuel, "Quantitative prediction of the modulation behavior of twisted nematic liquid crystal displays based on a simple physical model", Opt. Eng. 40, 2558-2564 (2001). DOI

[22]. R. C. Jones, “A new calculus for the treatment of optical systems”, J. Opt. Soc. Am. 31, 488-493 (1941). DOI

[23]. D. W. Berreman, "Optics in stratified and anisotropic media: 4x4-matrix formulation”, J. Opt. Soc. Am. 62, 502-510 (1972). DOI

[24]. J. E. Wolfe, R. A. Chipman, "Polarimetric characterization of liquid-crystal-on-silicon panels", Appl.Opt. 45, 1688-1703 (2006). DOI 
[25]. A. Márquez, I. Moreno, C. Iemmi, A. Lizana, J. Campos, M. J. Yzuel, "Mueller-Stokes characterization and optimization of a liquid crystal on silicon display showing depolarization", Opt. Express 16, 16691685 (2008). DOI

[26]. I. Moreno, A. Lizana, J. Campos, A. Márquez, C. Iemmi, M. J. Yzuel, “Combined Mueller and Jones matrix method for the evaluation of the complex modulation in a liquid-crystal-on-silicon display", Opt. Lett. 33, 627-629 (2008). DOI

[27]. N. G. Parke, “Optical algebra”, J. Math. Phys. 28, 131-139 (1949).

[28]. R. A. Chipman, "Polarimetry”, in Handbook of Optics, McGraw-Hill, New York (1995).

[29]. D. Goldstein, Polarized Light, Mercel Dekker, New York (2003).

[30]. A. Peinado, A. Lizana, J. Vidal, C. Iemmi, J. Campos, “Optimization and performance criteria of a Stokes polarimeter based on two variable retarders", Opt. Express 18, 9815-9830 (2010). DOI

[31]. P. Taylor, Theory and Applications of Numerical Analysis, Academic Press, London (1974).

[32]. A. De Martino, Y. Kim, E. Garcia-Caurel, B. Laude, B. Drévillon, “Optimized Mueller polarimeter with liquid crystals", Opt. Lett. 28, 616-618 (2003). DOI

[33]. D. S. Sabatke, M. R. Descour, E. L. Dereniak, W. C. Sweatt, S. A. Kemme, G. S. Phipps, “Optimization of retardance for a complete Stokes polarimeter", Opt. Lett. 25, 802-804 (2000). DOI

[34]. A. Lizana, N. Martin, M. Estapé, E. Fernández, I. Moreno, A. Márquez, C. Iemmi, J. Campos, M. J Yzuel, "Influence of the incident angle in the performance of liquid crystal on silicon displays", Opt. Express 17, 8491-8505 (2009). DOI

[35]. A. Lizana, A. Márquez, I. Moreno, C. Iemmi, J. Campos, M. J. Yzuel, "Wavelength dependence of polarimetric and phase-shift characterization of a liquid crystal on silicon display", J. Eur. Opt. Soc. Rap. Public. 3, 1-6 (2008). DOI

[36]. J. J. Gil, E. Bernabeu, “A depolarization criterion in Mueller matrices”, Opt. Acta/J. Mod. Opt. 32, 259261 (1985).

[37]. A. Lizana, I. Moreno, C. Iemmi, A. Márquez, J. Campos, M. J. Yzuel, "Time resolved Mueller matrix analysis of a liquid crystal on silicon display", Appl. Opt. 47, 4267-4274 (2008). DOI

[38]. R. Ossikovski, M. Anastasiadou, S. B. Hatit, E. Garcia-Caurel, A. De Martino, "Depolarizing Mueller matrices: How to decompose them?", Phys. Stat. Sol. A 205, 720-727 (2008). DOI

[39]. M. Foldyna, E. Garcia-Caurel, R. Ossikovski, A. De Martino, J. J. Gil, "Retrieval of a non-depolarizing component of experimentally determined depolarizing Mueller matrices", Opt. Express 17, 1279412806 (2009). DOI

[40]. S. Y. Lu, R. A. Chipman, "Interpretation of Mueller matrices based on polar decomposition", J. Opt. Soc. Am. A 13, 1106-1113 (1996). DOI

[41]. I. Moreno, P. Velásquez, C. R. Fernández-Pousa, M. M. Sánchez-López, F. Mateos, "Jones matrix method for predicting and optimizing the optical modulation properties of a liquid-crystal display", J. Appl. Phys. 94, 3697-3702 (2003). DOI

[42]. A. Lizana, I. Moreno, A. Márquez, C. Iemmi, E. Fernández, J. Campos, M. J. Yzuel, “Time fluctuations of the phase modulation in a liquid crystal on silicon display: characterization and effects in diffractive optics", Opt. Express 16, 16711-16722 (2008). DOI

[43]. I. Moreno, C. Iemmi, A. Márquez, J. Campos, M. J. Yzuel, “Modulation light efficiency of diffractive lenses displayed in a restricted phase-mostly modulation display", Appl. Opt. 43, 6278-6284 (2004). $\underline{\text { DOI }}$

[44]. A. Lizana, A. Márquez, L. Lobato, Y. Rodange, I. Moreno, C. Iemmi, J. Campos, “The minimum Euclidean distance principle applied to improve the modulation diffraction efficiency in digitally controlled spatial light modulators", Opt. Express 18, 10581-10593 (2010). DOI

[45]. A. Hermerschmidt, S. Osten, S. Krüger, T. Blümel, "Wave front generation using a phase-only modulating liquid-crystal based micro-display with HDTV resolution", Proc. SPIE 6584, 65840E (2007). DOI

[46].Z. Zhang, G. Lu, F. T. S Yu, "Simple method for measuring phase modulation in liquid crystal television", Opt. Eng. 33, 3018-3022 (1994). DOI 
[47]. J. R. Moore, N. Collings, W. A. Crossland, A. B. Davey, M. Evans, A. M. Jeziorska, M. Komarcevic, J. R. Parker, T. D. Wilkinson, H. Xu, "The silicon backplane design for an LCOS polarization-insensitive phase hologram SLM", IEEE Phot. Tech. Lett. 20, 60-62 (2008). DOI

[48]. J. A. Davis, I. Moreno, P. Tsai, "Polarization eigenstates for twisted-nematic liquid crystal displays", Appl. Opt. 37, 937-945 (1998). DOI

[49]. R. D. Juday, "Generality of matched filtering and minimum Euclidean distance projection for optical pattern recognition", J. Opt. Soc. Am A 18, 1882-1896 (2001). DOI

[50]. I. Moreno, J. Campos, C. Gorecki, M. J. Yzuel, "Effects of amplitude and phase mismatching errors in the generation of a kinoform for pattern recognition", Jap. J. Appl. Phys. 34, 6423-6432 (1995). DOI

[51].T. Fujita, H. Nishihara, J. Koyama, "Blazed gratings and Fresnel lenses fabricated by electron-beam lithography", Opt. Lett. 7, 578-580 (1982). DOI

\section{Introducción}

En las últimas décadas, las pantallas de cristal líquido (LCDs, Liquid Crystal Displays) han ganado un creciente protagonismo en las aplicaciones en óptica, debido a algunas de sus características técnicas. Por ejemplo, gracias a su enorme capacidad para modular espacialmente la fase de un haz de luz incidente, son extensamente utilizadas como moduladores espaciales de luz (SLM, Spatial Light Modulators) en numerosas aplicaciones [1-4].

Las pantallas de cristal líquido sobre silicio (LCoS, Liquid Crystal on Silicon) son un tipo particular de pantalla LCD que trabajan por reflexión. Gracias al doble paso que un haz de luz incidente realiza dentro de estos dispositivos, las pantallas LCoS presentan una mayor modulación de fase que una pantalla LCD con el mismo espesor y que opere en transmisión. Como consecuencia de esta ganancia en modulación de fase (entre otras interesantes características técnicas de las pantallas LCoS), muchos usuarios de pantallas LCD han adoptado esta nueva tecnología por reflexión, siendo hoy en día, un elemento clave en aplicaciones ópticas tales como: procesado óptico de imágenes [5-7], almacenamiento holográfico de datos [8], óptica adaptativa $[9,10]$, diagnóstico médico por imagen polarimétrica $[11,12]$, óptica difractiva [13-15], metrología óptica [16,17], entre otras.

Para garantizar la óptima utilización de las pantallas LCoS, resulta crucial disponer de un análisis completo de estos dispositivos, incluyendo información tan relevante como el desarrollo de métodos de caracterización y optimización válidos para trabajar con pantallas LCoS en función de todos los parámetros relevantes del sistema (ángulo de incidencia, longitud de onda operativa, etc.), análisis físicos del dispositivo para la determinación de un cuadro detallado de sus principales propiedades ópticas, la descripción cuantitativa de su operatividad y eficiencia en la aplicación final, etc.

Para cubrir dicha necesidad, en este trabajo se presenta un resumen de la tesis "Characterization and Optimization of Liquid Crystal on Silicon displays for their application to Diffractive Optics", la cual constituye un compendio de varios resultados enmarcados en el estudio de las pantallas LCoS. En particular, dicho trabajo representa un análisis completo de las pantallas LCoS, enfocándose especialmente para su aplicación en óptica difractiva. Por ello, pensamos que este trabajo puede constituir una herramienta de gran utilidad para todos aquellos posibles usuarios de estos dispositivos ópticos.

\section{Caracterización y optimización de las pantallas LCoS}

Para trabajar eficientemente con las pantallas LCDs, es indispensable tener un control preciso de la respuesta en amplitud y fase de las mismas. Para cubrir esta necesidad, existen en la literatura descripciones de diversos métodos de caracterización y optimización de las pantallas LCDs en transmisión [18-21]. Sin embargo, estos métodos de caracterización no son válidos para predecir la respuesta de las pantallas LCoS. En particular, los métodos previamente referenciados se basan en formalismos matemáticos, como el de Jones [22] o Berreman [23], que operan con las componentes vectoriales instantáneas del campo 
electromagnético. Dichas aproximaciones no son capaces de caracterizar haces de luz incluyendo cierto contenido en luz despolarizada o materiales despolarizantes, ya que los valores de despolarización se obtienen al promediar variaciones temporales (o espaciales) de las componentes instantáneas del campo electromagnético. En este sentido, varios autores han demostrado que al trabajar con las pantallas LCoS, cierto grado de despolarización, que depende de varios parámetros físicos del sistema, como el estado de polarización incidente o el nivel de gris enviado al modulador, puede añadirse al haz reflejado [24,25]. Debido a esta propiedad, para describir el comportamiento de una pantalla LCoS necesitamos algún método de caracterización alternativo basado en un formalismo matemático capaz de incluir en el modelo físico contribuciones de luz parcial o completamente despolarizada.

El objetivo de esta sección es la descripción completa de un método de caracterización y optimización valido para operar con las pantallas LCoS. El método aquí propuesto permite predecir con precisión la respuesta en amplitud y fase del modulador [25, 26]. La novedad de este método reside en la combinación de dos formalismos matemáticos ya existentes, el formalismo de Mueller-Stokes (M-S) [27] y el formalismo de Jones [22], permitiendo predecir la amplitud y fase del haz reflejado por el modulador, independientemente de su contenido en luz despolarizada.

\section{2.a. Predicción de la respuesta en amplitud del modulador}

Esta subsección presenta una técnica de caracterización, basada en los formalismos matemáticos de (M-S) y Jones, válida para predecir la respuesta en amplitud de una pantalla LCoS, y de forma más general, para cualquier tipo pantalla LCD, independientemente de su capacidad de despolarización. Un análisis detallado de esta técnica se puede encontrar en la Ref. [25].

El dispositivo estudiado es una pantalla Philips LCoS, modelo X97c3A0, comercializado por HoloEye Systems dentro del kit LC-R2500.
La pantalla tiene una resolución XGA (1024x768 pixeles), con un factor de llenado del 93\%. Los pixeles son cuadrados y están separados por una distancia, de centro a centro, de unos $19 \mu \mathrm{m}$. Con la pantalla apagada, el eje de anisotropía de las moléculas de cristal líquido presenta un giro a lo largo de la celda de unos 45 grados (es decir, es una pantalla de tipo twisted nematic). Además, el nivel de gris enviado al modulador está controlado digitalmente, pudiéndose enviar 256 niveles de gris distintos (8 bits).

El primer paso del modelo consiste en determinar la matriz de Mueller de la pantalla LCoS. El montaje experimental utilizado para tal fin se presenta en la Fig. 1. El montaje se ilumina con un láser de Helio-Neón, que genera un haz de luz linealmente polarizado, con una longitud de onda de $633 \mathrm{~nm}$. Detrás de la fuente de luz se coloca una lámina retardadora de media onda (Half-WP), que permite girar el plano de polarización a la salida del láser, y por tanto, controlar la intensidad de luz a la salida del polarizador lineal LP1. La pantalla LCoS, insertada entre el sistema generador de estados (PSG, Polarization State Generator) y el sistema detector de estados (PSD, Polarization State Detector), trabaja por reflexión, formando 4 grados entre el haz incidente y el reflejado. El PSG está formado por el polarizador lineal LP1, orientado paralelo a la vertical, seguido por una lámina retardadora de cuarto de onda (WP1). La lamina WP1 esta insertada en una plataforma rotatoria, pudiéndose así controlar su orientación electrónicamente. Variando la orientación de la lámina, se puede iluminar la pantalla LCoS con distintos estados de

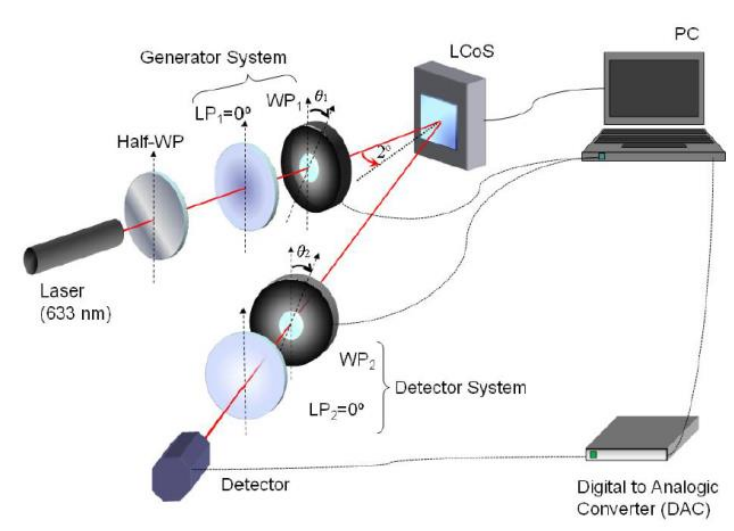

Fig. 1. Montaje experimental para la determinación de la matriz de Mueller de la pantalla LCoS. 
polarización. El PSD, que nos permite analizar el estado de polarización de la luz reflejada por el LCoS, está formado por los mismos elementos que el PSG, pero invirtiendo su orden. Finalmente, un radiómetro comercial (Newport 1830-C) situado a la salida del PSD se encarga de realizar las correspondientes medidas de intensidad a la salida del sistema.

La combinación del PSD y el radiómetro (brazo reflejado en Fig. 1) da lugar a un polarímetro completo de Stokes [28], capaz de determinar cualquier estado de polarización reflejado por la pantalla LCoS. Por tanto, dado que la matriz de Mueller $M$ de una muestra polarizante relaciona linealmente el estado de polarización de la luz incidente con el estado a la salida (reflejado, transmitido o disperso por la muestra) [28,29], mediante la generación de distintos estados de polarización con el PSG, y la correspondiente determinación del estado a la salida mediante el PSD, es posible diseñar un sistema de ecuaciones linealmente independiente que dé lugar a la determinación de los 16 coeficientes de la matriz de Mueller de la muestra. Para caracterizar completamente la matriz de Mueller se requieren, como mínimo, 4 estados de polarización linealmente independientes en la generación (determinados por 4 configuraciones del PSG) y 4 estados linealmente independientes en la detección (determinados por 4 configuraciones del PSD). La elección de estos estados no es única, y diferentes soluciones dan lugar a un polarímetro completo de Mueller, y por tanto, a la determinación completa de la matriz. Sin embargo, la calidad de la matriz de Mueller final depende en gran medida de los estados de polarización seleccionados [30]. Para estimar la validez de las configuraciones del PSG y PSD escogidas, es común utilizar diferentes criterios de calidad, como el cálculo del condicionamiento del sistema [31,32] o de sus varianzas [33].
En el presente trabajo, la determinación de la matriz de Mueller se ha realizado según el cálculo detallado en Ref. [34]. En concreto, se puede demostrar que para el montaje particular descrito en Fig. 1, la intensidad a la salida del PSD es [34]:

$$
\begin{aligned}
I\left(\theta_{2}\right)=\frac{1}{2}[ & S_{0}+S_{1} \cos ^{2}\left(2 \theta_{2}\right)+ \\
& \left.+S_{2} \sin \left(2 \theta_{2}\right) \cos \left(2 \theta_{2}\right)-S_{3} \sin \left(2 \theta_{2}\right)\right],
\end{aligned}
$$

donde $I$ solo depende de los parámetros de Stokes del haz incidente $\left(S_{0}, S_{1}, S_{2}\right.$ y $S_{3}$, que describen completamente el estado de polarización) y de la orientación $\theta_{2}$ de lámina retardadora en el PSD (WP2 en Fig. 1).

La ecuación (1) muestra como la intensidad a la salida del PSD es una función periódica respecto a $\theta_{2}$, pudiéndose descomponer como una suma de funciones sinusoidales cuyos argumentos son múltiples enteros de ?2. Por este motivo, se puede aplicar el método de detección síncrona [29], el cual permite estimar los coeficientes de la serie de Fourier representada en Ec. (1), siendo estos los distintos parámetros de Stokes del haz reflejado por la pantalla LCoS. En particular, realizando un sumatorio de diferentes intensidades correspondientes a $N$ valores de $\theta_{2}$ igualmente espaciados a lo largo de un periodo, algunos términos de Ec. (1) se reducen a cero debido a las propiedades de ortogonalidad de las funciones sinusoidales. Además, si se realiza un nuevo sumatorio de diferentes intensidades pero ahora, multiplicándolas por el coseno o el seno del ángulo correspondiente, son otros los términos de Ec. (1) que se anulan. Combinando estas informaciones, es posible encontrar la siguiente expresión para el vector de Stokes del haz reflejado [34]: 


$$
\left(\begin{array}{l}
S_{0} \\
S_{1} \\
S_{2} \\
S_{3}
\end{array}\right)=\left(\begin{array}{c}
2 \sum_{r=1}^{N} I\left(\frac{\pi}{2}, \theta_{2, r}\right)-4 \sum_{r=1}^{N} I\left(\frac{\pi}{2}, \theta_{2, r}\right) \cos \left(4 \theta_{2, r}\right) \\
8 \sum_{r=1}^{N} I\left(\frac{\pi}{2}, \theta_{2, r}\right) \cos \left(4 \theta_{2, r}\right) \\
8 \sum_{r=1}^{N} I\left(\frac{\pi}{2}, \theta_{2, r}\right) \sin \left(4 \theta_{2, r}\right) \\
-4 \sum_{r=1}^{N} I\left(\frac{\pi}{2}, \theta_{2, r}\right) \sin \left(2 \theta_{2, r}\right)
\end{array}\right)
$$

siendo $N$ el número de ángulos $\theta_{2}$ seleccionados y donde $\theta_{2, r}=2 \pi(r-1) / N$ con $r=1,2, \ldots, N$.

Según la Ec. (2), el estado de polarización del haz reflejado por la pantalla LCoS se puede determinar completamente a partir de diferentes medidas de intensidad correspondientes a diferentes orientaciones de la lámina WP2 (ver Fig. 1). El siguiente paso es ampliar esta capacidad de medida a la caracterización completa de la matriz de Mueller. Para ello, se aplica la técnica detallada en Ref. [34], donde se extiende el estudio anterior para un sistema combinado PSG-PSD. Para tal fin, se realizan diferentes medidas del vector de Stokes reflejado (según Ec. (2)) correspondientes a diferentes configuraciones del PSG, es decir, para diferentes orientaciones $\theta_{1}$ de la lámina WP1. Siguiendo este principio, se puede demostrar que la matriz de Mueller de un elemento polarizante puede escribirse de la siguiente forma:

$$
M=\frac{1}{N}\left(\begin{array}{llll}
\sum_{r=1}^{N} S_{0, r}-2 \sum_{r=1}^{N} S_{0, r} \cos \left(4 \theta_{1, r}\right) & 4 \sum_{r=1}^{N} S_{0, r} \cos \left(4 \theta_{1, r}\right) & 4 \sum_{r=1}^{N} S_{0, r} \sin \left(4 \theta_{1, r}\right) & 2 \sum_{r=1}^{N} S_{0, r} \sin \left(2 \theta_{1, r}\right) \\
\sum_{r=1}^{N} S_{1, r}-2 \sum_{r=1}^{N} S_{1, r} \cos \left(4 \theta_{1, r}\right) & 4 \sum_{r=1}^{N} S_{1, r} \cos \left(4 \theta_{1, r}\right) & 4 \sum_{r=1}^{N} S_{1, r} \sin \left(4 \theta_{1, r}\right) & 2 \sum_{r=1}^{N} S_{1, r} \sin \left(2 \theta_{1, r}\right) \\
\sum_{r=1}^{N} S_{2, r}-2 \sum_{r=1}^{N} S_{2, r} \cos \left(4 \theta_{1, r}\right) & 4 \sum_{r=1}^{N} S_{2, r} \cos \left(4 \theta_{1, r}\right) & 4 \sum_{r=1}^{N} S_{2, r} \sin \left(4 \theta_{1, r}\right) & 2 \sum_{r=1}^{N} S_{2, r} \sin \left(2 \theta_{1, r}\right) \\
\sum_{r=1}^{N} S_{3, r}-2 \sum_{r=1}^{N} S_{3, r} \cos \left(4 \theta_{1, r}\right) & 4 \sum_{r=1}^{N} S_{3, r} \cos \left(4 \theta_{1, r}\right) & 4 \sum_{r=1}^{N} S_{3, r} \sin \left(4 \theta_{1, r}\right) & 2 \sum_{r=1}^{N} S_{3, r} \sin \left(2 \theta_{1, r}\right)
\end{array}\right),
$$

donde $\theta_{1, r}=2 \pi(r-1) / N$ siendo $N$ el número de estados de polarización generados con el PSG, y el valor $r(r=1,2, \ldots, N)$ un parámetro que indexa el término particular de la suma.

La Ec. (3) indica que generando adecuadamente varios estados de polarización (mínimo 4) correspondientes a diferentes orientaciones $\theta_{1}$ de la lámina WP1, y midiendo los correspondientes estados reflejados según (2), la matriz de Mueller de un elemento polarizarte queda completamente determinada.
Finalmente, se verifica experimentalmente el desarrollo de caracterización propuesto en esta sección. En particular, se ha medido, según Ec. (3), la matriz de Mueller experimental del prototipo LCoS descrito anteriormente en esta sección. El dispositivo se ha medido para diferentes niveles de gris (de 0 a 255 en pasos de 20). La validez de los resultados se analiza estudiando la capacidad de la matriz de Mueller medida para predecir, dado un estado de polarización incidente, el correspondiente estado de polarización reflejado. 
Los resultados obtenidos constatan la validez del presente método de caracterización. Como ejemplo, en la Fig. 2 se muestran los parámetros de Stokes del haz reflejado por la pantalla LCoS en función del nivel de gris, cuando el dispositivo es iluminado con un estado de polarización lineal (orientado a 135 grados de la vertical). Las líneas continuas representan los valores teóricos (calculados multiplicando el vector de Stokes teórico incidente por la matriz de Mueller experimental de la pantalla LCoS) y los símbolos representan los datos experimentales (medidos según la relación dada en Ec. (2)).

Se observa un excelente acuerdo entre la teoría y las medidas experimentales para todos los parámetros de Stokes. Este acuerdo se verifica para cualquier estado de polarización incidente, poniendo en valor la capacidad del método para predecir el estado de polarización reflejado por la pantalla, y por tanto, la respuesta en intensidad del mismo. En efecto, está última se calcula fácilmente como la proyección del estado de polarización reflejado sobre una configuración particular elegida para el PSD del sistema.

Finalmente cabe destacar que en función de la aplicación donde la pantalla LCoS es requerida, puede ser necesario modificar alguno de los parámetros físicos fijados en el anterior ejemplo, como por ejemplo, el ángulo de incidencia o la longitud de onda de la fuente de iluminación. En ese caso, la respuesta del modulador puede ser significativamente diferente, y por consiguiente, su matriz de Mueller será distinta. No obstante, la robustez

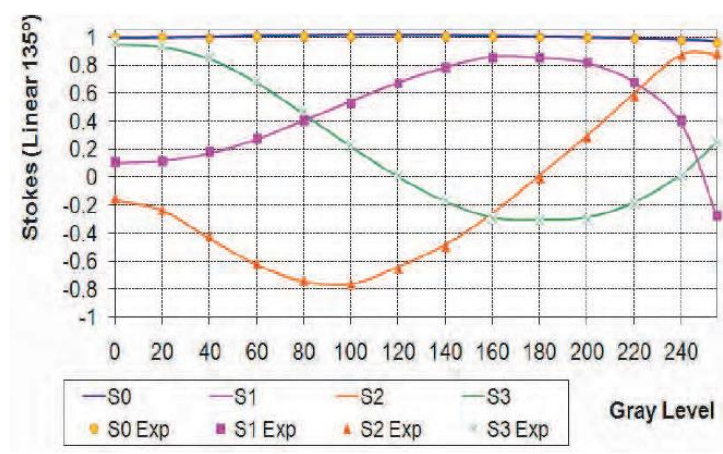

Fig. 2. Parámetros de Stokes en función del nivel de gris para un estado de polarización incidente a 135 grados de la vertical del laboratorio. del método de caracterización descrito en esta sección permite extenderlo fácilmente a diferentes condiciones experimentales $[34,35]$.

\section{2.b. Predicción de la respuesta en fase del modulador}

En esta subsección, el método de caracterización de la respuesta en amplitud de pantallas LCDs, presentado en la sección 2.a., se extiende también a la predicción de su respuesta en fase. Para tal fin, como la matriz de Jones de un elemento polarizante contiene información pura sobre su capacidad para modular la fase de un haz de luz incidente, en este trabajo se propone una conversión de la matriz de Mueller experimental del dispositivo, determinada según 2.a., a la correspondiente matriz de Jones del retardador equivalente.

No todas las matrices de Mueller tienen su equivalente matriz de Jones, siendo una condición necesaria y suficiente para tal fin que la matriz de Mueller cumpla el llamado criterio de la traza o teorema de Gil-Bernabeu [36]. Como caso particular y de interés para nuestros propósitos, cabe destacar que las matrices de Jones no son válidas para describir la capacidad despolarizante de un elemento polarimétrico. Sin embargo, como se ha comentado anteriormente, las pantallas LCoS pueden introducir cierta cantidad de luz despolarizada al haz de luz reflejado [24,25,37]. Por ello, antes de realizar la conversión entre la matriz de Mueller de una pantalla LCoS y su equivalente matriz de Jones, es necesario realizar una descomposición de la información incluida en la matriz de Mueller. De este modo, puede ser desgranada en diferentes matrices de Mueller de elementos polarimetricos básicos, y así, su contenido puro en fase puede ser extraído. Llegados a este punto, una vez la matriz de Mueller del retardador puro es obtenida mediante una descomposición matricial, es posible convertir dicha matriz del retardador en su correspondiente matriz de Jones.

La elección del método de descomposición de la matriz de Mueller no es única, existiendo diferentes técnicas que pueden ser aplicadas para este fin [38,39]. En particular, en este trabajo se ha utilizado la descomposición matricial de Lu-Chipman [40], la cual describe 
cómo la matriz de Mueller de un elemento polarimétrico puede descomponerse como el producto de tres matrices de Mueller más básicas: la matriz de Mueller de un diatenuador puro $M_{\mathrm{D}}$, la matriz de Mueller de un retardador puro $M_{\mathrm{R}}$ y la matriz de Mueller de un despolarizador $M_{\Delta}$. En el caso particular de las pantallas LCoS, diferentes estudios han demostrado que son elementos no diatenuantes $[25,34,35]$, pudiéndose aproximar la matriz MD de la descomposición matricial a la matriz identidad. Teniendo esto en cuenta, al aplicar la descomposición de Lu-Chipman a la matriz de Mueller de la pantalla LCoS, se obtiene la siguiente relación [26]:

$$
M_{L C o S}=M_{\Delta} \cdot M_{R}=\left(\begin{array}{cc}
1 & \overrightarrow{0}^{T} \\
\vec{P}_{\Delta} & m_{\Delta}
\end{array}\right) \cdot\left(\begin{array}{cc}
1 & \overrightarrow{0}^{T} \\
\overrightarrow{0} & m_{R}
\end{array}\right),
$$

donde $\quad \overrightarrow{0}^{T}=\left(\begin{array}{llll}0 & 0 & 0 & 0\end{array}\right), \quad \vec{P}_{\Delta} \quad$ es el vector polarizancia [29], y donde las matrices $m_{\Delta}$ y $m_{R}$ son dos submatrices $3 \times 3$ cuyos coeficientes se calculan según el método detallado en Ref. [29].

Una vez se ha calculado la matriz de Jones del retardador equivalente a partir de la matriz de Mueller del retardador $M_{R}$, es posible aplicar una metodología de evaluación de la modulación de fase compleja. En particular, se utiliza la técnica propuesta en Ref. [41], la cual ha demostrado ser eficaz para predecir la respuesta de fase en dispositivos operando en transmisión. En particular, en Ref. [41] se deduce una expresión formal para describir la fase total $\delta$ de una pantalla LCD situada entre dos polarizadores con orientaciones $\theta_{1}$ y $\theta_{2}$ [41]:

$$
\begin{aligned}
\delta & =\beta+\delta_{M}=\beta+ \\
& +\arctan \left(\frac{A_{I} \cos \left(\theta_{1}+\theta_{2}\right)+B_{I} \sin \left(\theta_{1}+\theta_{2}\right)}{A_{R} \cos \left(\theta_{1}-\theta_{2}\right)+B_{R} \sin \left(\theta_{1}-\theta_{2}\right)}\right)
\end{aligned}
$$

donde $\beta$ es una fase externa que depende del nivel de gris aplicado al modulador $[26,41]$, y donde la contribución de fase $\delta_{M}$ depende de las orientaciones $\theta_{1}$ y $\theta_{2}$ de los polarizadores, así como de los coeficientes complejos $A_{I}, B_{I}, A_{R}$ y $B_{R}$ (que dependen del voltaje aplicado).

A partir de la matriz de Mueller del retardador $M_{R}$ y de las transformaciones dadas en la Ref. [29], los coeficientes $A_{I}, B_{I}, A_{R}$ y $B_{R}$ se pueden determinar en función del nivel de gris. Por tanto, dado un par de orientaciones $\theta_{1}$ y $\theta_{2}$ conocidas, se puede calcular la fase $\delta_{M}$. Sin embargo, no se tiene ninguna información a priori de la fase externa $\beta$. Por ello, se realizan medias experimentales de la fase total $\delta$ en función del nivel de gris (utilizando el método de medición de fase descrito en [42]), y a partir de ellas, se calcula la fase $\beta$ en función del nivel de gris, despejándola de Ec. (5), dadas unas orientaciones $\theta_{1}$ y $\theta_{2}$ conocidas. Finalmente, puesto que la fase externa $\beta$ no depende del estado de polarización generado con el PSG, o del detectado con el PSD, una vez la fase $\beta$ ha sido calibrada, la respuesta de modulación de fase de la pantalla LCoS en función del nivel de gris puede ser completamente predicha siguiendo Ec. (5).

\section{2.c. Optimización de la respuesta en amplitud y fase de la pantalla LCoS}

En muchas aplicaciones de las pantallas LCDs dentro el campo de la óptica difractiva, la eficiencia de los elementos difractivos es máxima cuando operan como moduladores solo de fase [43]. En dichas aplicaciones es muy interesante operar con una modulación de fase lineal que alcance los 360 grados, acompañada de una amplitud de valor constante en función del nivel de gris.

Siguiendo los métodos de caracterización descritos anteriormente, se puede predecir la respuesta de amplitud (sección 2.a) y fase (sección 2.b) de las pantallas LCoS. Tomando ventaja de esta capacidad de predicción, en esta sección se propone un método de optimización de la respuesta de las pantallas LCoS útil para maximizar la eficiencia de aquellos elementos difractivos generados con dichos dispositivos.

El método de optimización propuesto en esta sección se basa en un algoritmo de búsqueda numérica que permite determinar parejas de estados de polarización, para la incidencia y la detección, óptimos para el uso de las pantallas LCoS en el régimen de modulación solo en fase. Partiendo de un par de estados de polarización iniciales, el algoritmo de búsqueda proporciona un par de estados de polarización solución, que maximizan cierta figura de mérito $Q$. El método permite que cualquier estado de polarización pueda ser solución del sistema de búsqueda, y no está restringido únicamente a 
estados de polarización lineales, como sucede en numerosos protocolos de optimización. En este sentido, se ha aplicado un sistema de búsqueda de estados de polarización más general, ya que esto permite la obtención de mejores resultados en términos de modulación solo de fase [25].

En este trabajo, la figura de mérito $Q$ utilizada para optimizar la respuesta solo de fase de la pantalla LCoS, y que se muestra a continuación, se ha diseñado partiendo de tres premisas: mínimo valor de la transmitancia lo más grande posible, máxima modulación de fase y mínimo contraste de transmitancia.

$$
Q=\frac{1}{\lambda_{1}+\lambda_{2}+\lambda_{3}}\left(\lambda_{1} \frac{\Delta \tau}{2 \pi}+\lambda_{2}(1-\Delta T)+\lambda_{3} T_{m}\right),
$$

donde $\Delta \tau$ es la máxima variación de fase, $\Delta T$ es la máxima variación de la transmitancia y $T_{m}$ denota el mínimo valor de la transmitancia. Además, el peso específico de estos tres parámetros físicos dentro del proceso numérico puede detallarse variando la relación entre los coeficientes $\lambda_{1}, \lambda_{2}$ y $\lambda_{3}$.

Maximizando la figura de mérito $Q$ descrita en Ec. (6), se han obtenido diferentes configuraciones del PSG y PSD las cuales proporcionan excelentes resultados experimentales en términos de respuesta solo de fase [26]. Como ejemplo, la Fig. 3 muestra los resultados para la intensidad (eje de la izquierda, en rosa) y la fase (eje de la derecha, en marrón) en función del nivel de gris, correspondientes a una solución hallada por el programa de optimización antes descrito (los datos numéricos para las orientaciones de polarizadores y láminas en los sistemas PSG y PSD pueden encontrarse en [25]). Mientras que

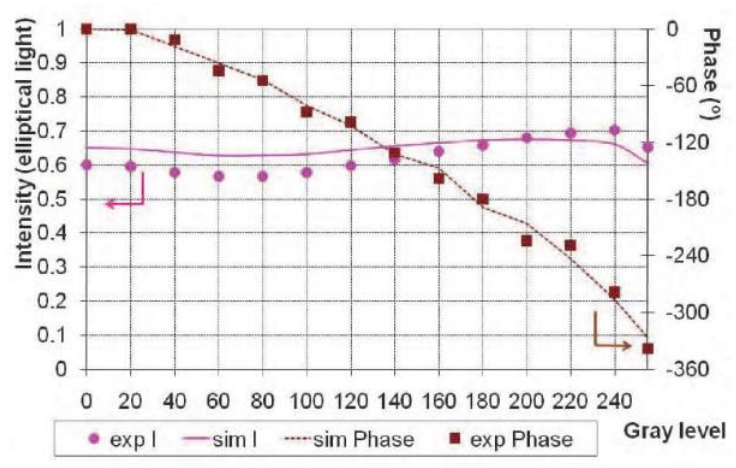

Fig. 3. Respuesta solo en fase de la pantalla LCoS. las líneas continuas describen las predicciones teóricas, los símbolos representan las medidas experimentales (obtenidas según el método de medida de fase descrito en [42]). Se observa un excelente acuerdo entre predicción teórica y medida experimental, además de un excelente resultado en términos de modulación solo en fase. En particular, en la Fig. 3 se puede observar un valor de la intensidad prácticamente continuo (en torno al valor 0.65) en función del nivel de gris, acompañado de una modulación de fase cercana a los 360 grados para una longitud de onda de $633 \mathrm{~nm}$.

\section{Fluctuaciones temporales de la fase: efectos en la eficiencia de elementos ópticos difractivos}

En esta sección se presentan evidencias experimentales del fenómeno físico de las fluctuaciones temporales de la fase presentes al trabajar con las pantallas LCoS [42,44], así como

un estudio sobre sus efectos en la generación de elementos ópticos difractivos. Este fenómeno, que puede degradar significativamente la eficiencia de los elementos difractivos generados por dichas pantallas [42], se origina en el tipo de esquemas eléctricos utilizados por estos dispositivos para determinar los diferentes niveles de gris [45].

\section{3.a. Evidencia experimental de las fluctuaciones temporales de la fase en pantallas LCos}

Para medir empíricamente el fenómeno de las fluctuaciones de fase, se ha utilizado el montaje experimental, basado en el fenómeno de la difracción, que se muestra en la Fig. 4. Como fuente de iluminación, se utilizada un haz de luz puntual laser (He-Ne, $633 \mathrm{~nm}$ ), que ilumina la pantalla LCoS con un ángulo de incidencia de 2 grados. Los sistemas generador y detector de estados de polarización (PSG y PSD respectivamente), son los mismos que los utilizados en el montaje experimental presentado en Fig. 1. Para asegurar una utilización de la pantalla $\mathrm{LCoS}$ en el régimen de modulación solo de fase, se utilizada la configuración de PSG y PSD dada en [25]. A 
continuación, se envía al modulador una red binaria de fase, y mediante dos radiómetros (Newport 1830-C) situados en el plano difractivo lejano, se registran las intensidades de los correspondientes órdenes de difracción cero $\mathrm{y}$ uno. Finalmente, las dos medidas radiométricas se sincronizan en un osciloscopio (Tektronix TDS3012B), permitiendo realizar medidas de la intensidad de los diferentes órdenes de difracción en función del tiempo.

Utilizando el montaje detallado en la Fig. 4, se ha medido la intensidad en función del tiempo de los ordenes cero y uno, correspondientes a tres redes binarias con distintas combinaciones de niveles de gris: $(0,120),(0,211)$ y $(0,255)$. En todos los casos se observa una fluctuación de la intensidad de los diferentes órdenes de difracción en función del tiempo, con una amplitud de la fluctuación cuyo valor depende del nivel de gris enviado a la pantalla LCoS [42].

Llegados a este punto, es posible deducir una relación analítica para las intensidades de los ordenes cero y uno en función de la diferencia de fase $\Phi$ entre los dos niveles de gris de una red binaria [46]. En particular, para una estructura periódica con dos niveles del mismo tamaño (ciclo de trabajo del 50\%), las intensidades normalizadas en función del tiempo, para los órdenes 0 y \pm 1 pueden escribirse como sigue:

$$
I_{0}(t)=\frac{1}{2}(1+\cos \Phi(t))
$$

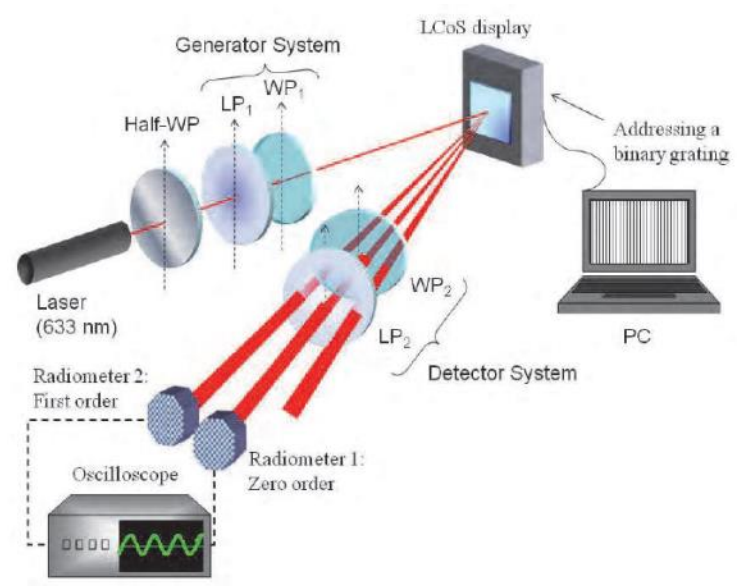

Fig. 4. Montaje experimental basado en el fenómeno de la difracción para la medida de la modulación de fase en función del tiempo.

$$
I_{ \pm 1}(t)=\frac{1}{\pi^{2}}(1-\cos \Phi(t)) .
$$

De las relaciones dadas en Ecs. (7) y (8), se puede deducir una expresión para la modulación de fase en función del tiempo:

$$
\Phi(t)=\frac{4 I_{0}(t)-\pi^{2} I_{1}(t)}{4 I_{0}(t)+\pi^{2} I_{1}(t)} .
$$

En la Fig. 5 se presentan los resultados para la diferencia de fase $\Phi$ entre dos niveles de gris en función del tiempo. Los resultados mostrados se han obtenido a partir de la Ec. (9), introduciendo los valores de las intensidades de los ordenes $0 \mathrm{y}$ \pm 1 correspondientes a las redes binarias con los niveles de gris $(0,120)$, en negro, $(0,211)$, en rojo, y $(0,255)$, en azul.

Los resultados presentados en la Fig. 5 muestran como en las pantallas LCoS, la diferencia de fase asociada a dos niveles de gris distintos no corresponde a un valor constante, sino que varía en función del tiempo. Por tanto, estos resultados representan una evidencia experimental del fenómeno de las fluctuaciones de la fase en función del tiempo. También se observa claramente que la amplitud de dichas fluctuaciones depende de los niveles de gris utilizados, pudiéndose alcanzar valores significativamente grandes, como es el caso de la red binaria $(0,211)$, donde una fase promedio de unos 180 grados está acompañada de unas fluctuaciones de fase con una amplitud de unos 60 grados (curva en rojo).

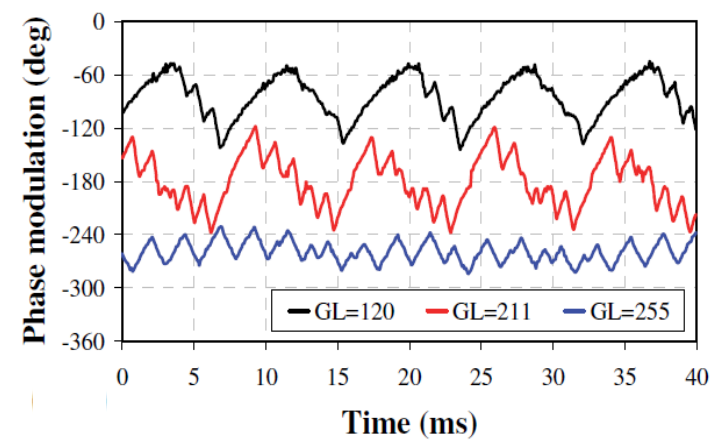

Fig. 5. Fluctuaciones temporales de la fase correspondiente a fases binaras con niveles de gris: 0-120 (en negro), 0-221 (en rojo) y 0-255 (en azul). 


\section{3.b. Efecto de la fluctuaciones de fase sobre la eficiencia en elementos ópticos difractivos}

En esta subsección se discute el efecto de las fluctuaciones temporales de la fase observada en las pantallas LCoS en la eficiencia de los elementos ópticos difractivos generados con estas pantallas. Se ha observado que en presencia de fluctuaciones de fase significativas, la eficiencia de los elementos ópticos difractivos puede degradarse. Como ejemplo de este hecho, se muestra el efecto de dichas fluctuaciones sobre un holograma digital binario: el holograma binario de una mariposa con un cambio de fase ? entre sus dos niveles de gris igual a 180 grados.

Para este estudio, se utiliza la pantalla LCoS con la configuración de modulación solo de fase correspondiente a los datos mostrados en Fig. 3. Mediante la combinación de un filtro espacial y una lente convergente, se genera un haz extenso y colimado que ilumina toda el área de la pantalla LCoS. Finalmente, una segunda lente

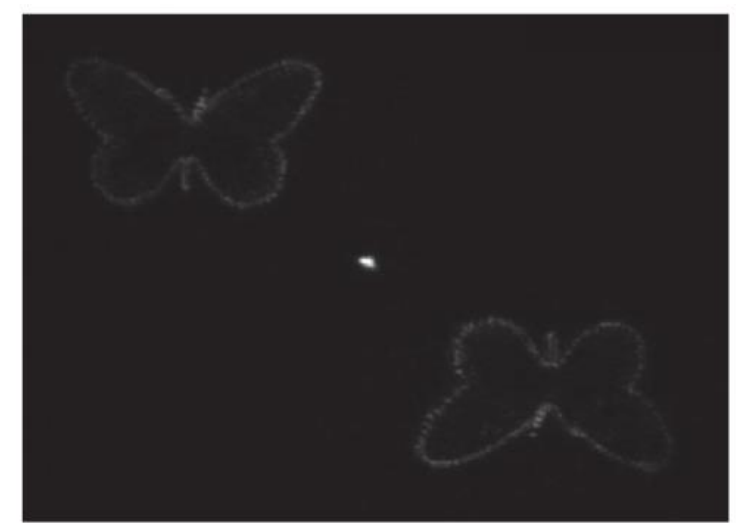

Fig. 6. Holograma digital diseñado para reconstruir una mariposa.

convergente situada en el haz reflejado, produce la imagen del infinito sobre una cámara CCD dispuesta en su plano focal. De este modo, al enviar la información de fase de un holograma digital sobre la pantalla LCoS, se obtiene sobre la cámara CCD su espectro de Fourier, obtenido por difracción. Una vez el montaje está dispuesto, se ha enviado un holograma de fase binario ideado para reconstruir una mariposa. La fase promedio entre niveles de gris es igual a 180 grados. Con el objetivo de separar espacialmente la reconstrucción de los diferentes órdenes de difracción, se ha añadido también una fase lineal a lo largo de la dirección diagonal.

Los resultados obtenidos se muestran en Fig. 6, donde el holograma de la mariposa se reconstruye en los ordenes \pm 1 . Teóricamente, al enviar un holograma binario con una diferencia de fase igual a 180 grados, en el orden 0 se debería obtener una valor de intensidad nulo [42]. Sin embargo, se observa como aparece un punto luminoso situado en el eje óptico. Esto es debido a que la fase está fluctuando en función del tiempo en torno al valor medio de 180 grados, y solo en esos instantes, donde la fase es exactamente igual a cero, la intensidad del orden cero es nula. Para los demás instantes de tiempo, al producirse en la fase una cierta desviación del valor teórico de 180 grados, se registra cierto valor de intensidad no nulo en el orden 0 . Finalmente, puesto que la CCD reproduce una imagen resultado de un promedio temporal, se obtiene un orden 0 no nulo, probando una degradación en la eficiencia del elemento difractivo final.

\section{Uso del principio de Mínima Distancia Euclidiana para aumentar la eficiencia de hologramas digitales en presencia de fluctuaciones temporales de la fase}

Algunos modelos de pantallas LCoS permiten enviar diferentes tipos de secuencias eléctricas para el control de los niveles de gris del dispositivo. Estas diferentes secuencias eléctricas dan lugar a distintas respuestas y eficiencias del modulador [45,47]. En particular, se ha observado experimentalmente una tendencia que muestra que aquellas secuencias eléctricas que proporcionan una mayor modulación de fase también tienden a tener asociadas una mayor amplitud de las fluctuaciones de la fase [44]. Sin embargo, para tener una eficiencia lo mayor posible al generar elementos ópticos difractivos con las pantallas LCoS, es importante, por un lado, disponer de una modulación de fase lo mayor posible (generalmente de unos 360 grados), y por otro, una amplitud de la fluctuación de la fase lo menor posible [44]. Por ello, es importante 
llegar a una relación óptima entre una modulación de fase lo mayor posible (aumenta la eficiencia de los elementos ópticos difractivos) y una amplitud de las fluctuaciones de fase lo menor posible (evita degradar la eficiencia de los mismos).

En esta subsección se presenta una técnica de mapeado de fase que permite maximizar la eficiencia final de los hologramas digitales generados con la pantalla LCoS al utilizar secuencias eléctricas con una modulación de fase menor de 360 grados (y por tanto, menor amplitud de la fluctuación).

\section{4.a. Esquemas de codificación de fase: Mapeado lineal y mapeado saturado}

Para implementar con exactitud hologramas digitales mediante el uso de pantallas LCD, es muy importante generar experimentalmente una distribución de fase tan cercana como sea posible a la diseñada teóricamente. Sin embargo, debido a diferentes no-linealidades, presentes en la implementación experimental, es imposible alcanzar la distribución de fase ideal. Para reducir las fuentes de degradación experimentales en la implementación de hologramas digitales, algunos autores han propuesto varias estrategias [21,48]. Por ejemplo, si el rango dinámico de modulación de fase proporcionado por la pantalla LCD es menor que 360 grados, se puede aplicar el principio de Mínima Distancia Euclidiana [49,50]. De esta forma, si se selecciona un mapeado de fase apropiado para la implementación de la fase, la eficiencia difractiva puede verse significativamente aumentada [50].

A continuación se revisan dos esquemas de mapeado de fase: el mapeado lineal y el mapeado saturado. Estos dos esquemas se representan en Fig. 7, donde la fase que realmente se implementa experimentalmente (indicada con el símbolo $p$ ), se representa en función de la fase que idealmente se quiere enviar al LCD (símbolo $\varphi$ ). Cabe destacar que los valores de la diagonal, representados con una línea negra y discontinua, representan el caso en que la fase $(\varphi)$ diseñada para el LCD es exactamente igual a la fase $(p)$ que realmente se implementa (caso ideal).
Supongamos ahora que los valores de fase que realmente podemos disponer mediante el uso de la pantalla LCD están dentro del rango (0, $\varepsilon)$, con $\varepsilon<2 \pi$. En esta situación, los valores de fase del mapeado lineal vendrían dados por la línea roja representada en la Fig. 7 (modelo 1). Una alternativa a este esquema es el mapeado saturado, representado en azul en la Fig. 7, (modelo 2). Este segundo esquema representa un codificado de fase más eficiente. En particular, el modelo 2 representa el emparejamiento perfecto entre $(p)$ y $(\varphi)$ hasta el valor de fase $\varphi=\varepsilon$. A partir de este valor, se realiza un saturado de la fase, dando a cada valor $\varphi>\varepsilon$ el valor de fase más cercano en el dominio de modulación de fase, según el criterio de la mínima distancia Euclidiana.

\section{4.b. Resultados experimentales}

En esta sección se compara la eficiencia de un elemento difractivo típico, una red Blazed [51], al ser generada con una pantalla LCoS cuando se utilizan las tres secuencias distintas (secuencias \#1, \#2 y \#3) estudiadas en Ref. [44]. Cabe destacar que la modulación de fase que proporcionan las secuencias \#1 y \#2 es aproximadamente de 360 grados, pero que la modulación que proporciona la secuencia \#3 no alcanza este valor, siendo aproximadamente de unos 280 grados [44]. Por ello mismo, el valor para la amplitud de las fluctuaciones de la fase correspondiente a la secuencia \#3 es significativamente menor que para las secuencias \#1 y \#2 [44].

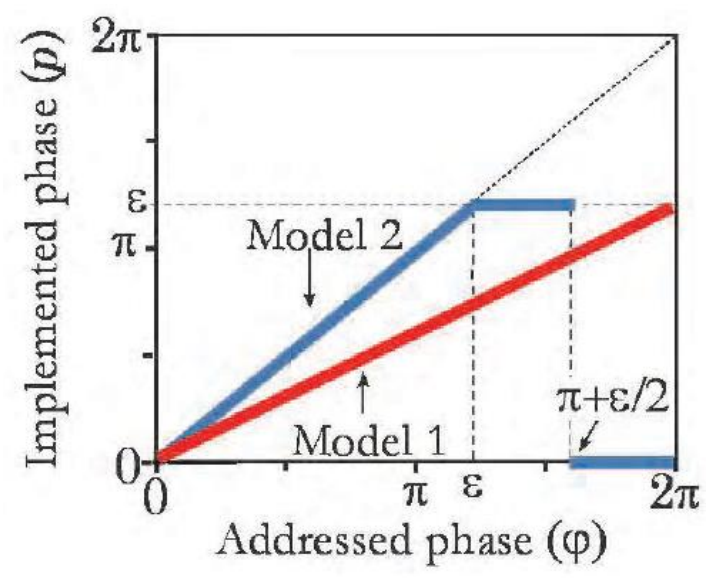

Fig. 7. Esquema de mapeado de fase: mapeado lineal (Modelo 1 , en rojo); mapeado saturado (modelo 2, en azul). 

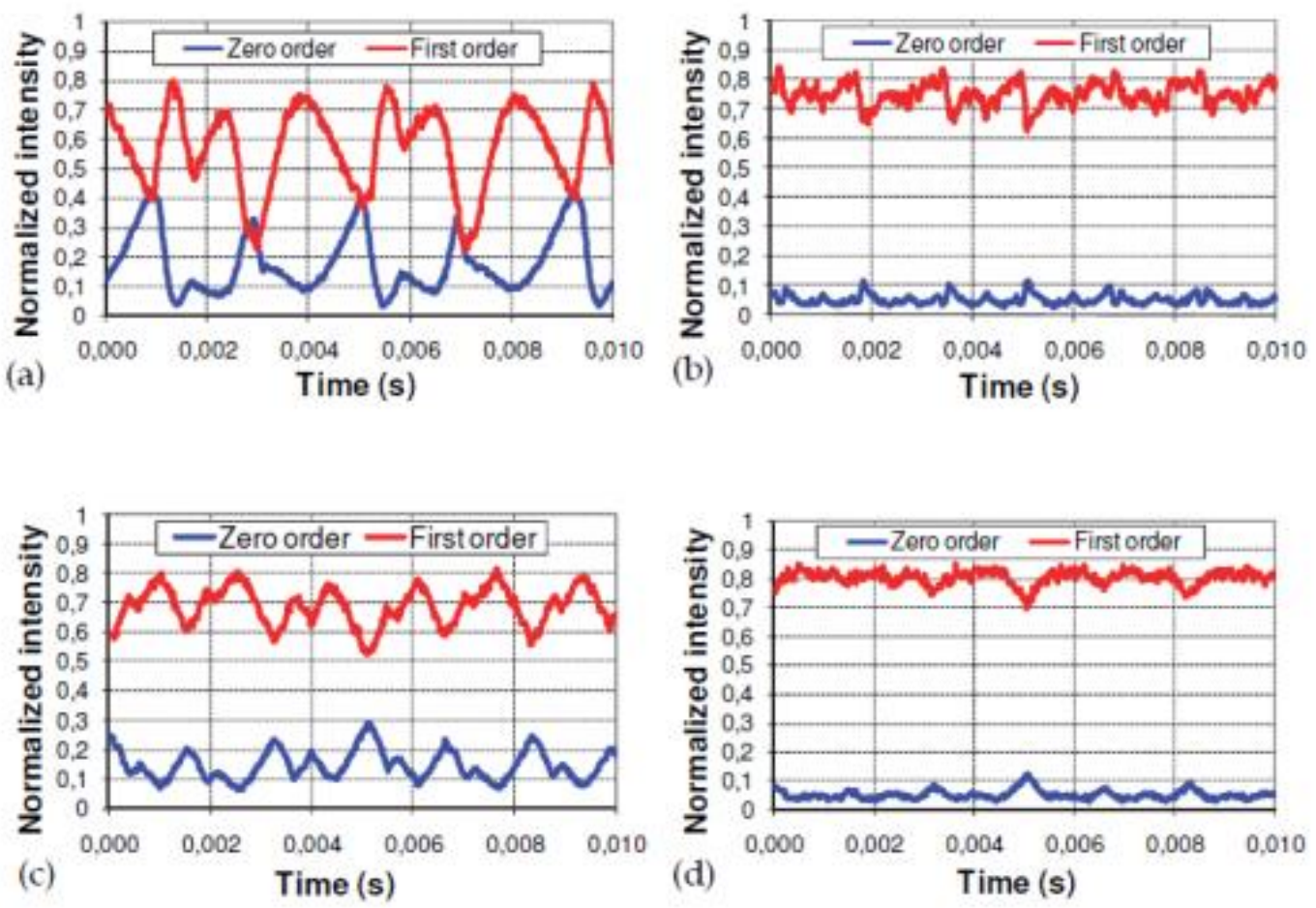

Fig. 8. Intensidad de los órdenes de difracción 0 y 1 obtenidos al generar una red Blazed con la secuencia eléctrica: (a) \#1, (b) \#2, (c) \#3 con mapeado lineal, y (d) \#3 con mapeado saturado.

Con una pantalla LCoS se ha implementado experimentalmente una red Blazed para las secuencias \#1, \#2 y \#3, y en todos los casos, se ha utilizado el mapeado lineal como codificación de fase. Además, como la modulación de fase correspondiente a la secuencia \#3 es menor de 360 grados, con esta secuencia, también se ha aplicado el mapeado saturado explicado en la sección 4.a.

Utilizando el montaje experimental dado en Fig. 4. e iluminando el sistema con un laser He$\mathrm{Ne}(633 \mathrm{~nm})$, se ha medido la intensidad en función del tiempo de los órdenes de difracción $0 \mathrm{y} \pm 1$ correspondientes a las diferentes redes Blazed implementadas. El periodo de las redes se ha fijado a 16 píxeles, siendo un valor suficiente para permitir despreciar el efecto de cuantificación de los niveles de fase.

Los resultados obtenidos se muestran en la Fig. 8. Las Figs. 8(a), 8(b) y 8(c) muestran las intensidades en función del tiempo para los órdenes de difracción 0 (en azul) y 1 (en rojo) correspondientes a las secuencias eléctricas \#1, \#2 y \#3 respectivamente, en todos los casos, utilizando un mapeado lineal. Por su parte, la figura 8(d) muestra los mismos resultados, pero para la secuencia \#3 cuando se usa un mapeado saturado.

Mientras que la mayor amplitud de las fluctuaciones de la intensidad se observa para la secuencia eléctrica \#1 (Fig. 8(a)), la mejor eficiencia difractiva se obtiene para la secuencia \#3 cuando se le aplica en mapeado saturado (Fig. 8(d)). Por lo tanto, aunque la secuencia \#3 proporciona un rango de modulación de fase menor de 360 grados, al aplicarle un esquema de codificación de fase eficiente (el mapeado saturado), representa la configuración más estable $y$ eficiente para generar elementos difractivos con la pantalla LCoS.

\section{Conclusiones}

Este trabajo presenta un estudio exhaustivo de las pantallas LCoS para su aplicación en la generación de elementos ópticos difractivos. Por ello, el análisis aquí detallado puede resultar de gran utilizad, así como de guía, para usurarios de 
la tecnología LCoS, permitiendo maximizar la eficiencia de estos dispositivos en diversas aplicaciones, como puede ser en aplicaciones para holografía digital.

En particular, este estudio presenta un método de caracterización y optimización de pantallas LCoS, basado en una combinación entre los formalismos matemáticos de Mueller y Jones, que permite optimizar la respuesta en amplitud $y$ fase del modulador independientemente de los efectos de despolarización presentes en estos dispositivos. Además, se han proporcionado evidencias experimentales del fenómeno de las fluctuaciones de fase en función del tiempo, demostrando que pueden degradar la respuesta de las pantallas LCoS. En este sentido, también se ha presentado una discusión sobre el efecto desfavorable de estas fluctuaciones en la generación de hologramas digitales, donde se destaca la importancia de encontrar secuencias eléctricas para cargar en los LCoS que permitan obtener un equilibrio entre dos parámetros directamente relacionados: el rango de modulación de fase (mayor eficiencia de los hologramas digitales para mayor modulación de fase) y la amplitud de las fluctuaciones de fase (mayor eficiencia para menor valor de la amplitud de fluctuación).

Finalmente, con la intención de minimizar el efecto de las fluctuaciones de fase en la generación de hologramas digitales, también se ha propuesto un método de codificación de fase para la implementación de los hologramas. Además, se ha demostrado experimentalmente la validez de esté método de codificación en términos de eficiencia de los elementos ópticos difractivos implementados en las pantallas LCoS.

\section{Agradecimientos}

Se agradece el soporte económico del Ministerio de Educación y Ciencia de España (FIS20913955-C02-01 and FIS209-13955-C02-02). 